\title{
MouseTrace: A better mousetrap for catching decision processes
}

\author{
J. D. JASPER and JENNIFER SHAPIRO \\ University of Toronto, Toronto, Ontario, Canada
}

\begin{abstract}
This paper describes MouseTrace, a computer program designed to monitor the process by which people make decisions by presenting complex choice information to subjects in the form of an alternativeby-attribute matrix. MouseTrace is a Windows-based extension of another program called MouseLab; however, MouseTrace accommodates significantly more information and allows for multiple responses, as well as multiple decision stages. It is also easier to use for both subjects and experimenters and more sophisticated in terms of its features and the data it records. These data include what information was examined, when, and in what order. This information can be used to derive a variety of cognitive process measures, including those related to search depth, sequence, and content, which in turn can be used to provide unique insights into decision-making theory, individual differences, and a variety of decision variables.
\end{abstract}

The area of decision making can be broadly lumped into two methodologically distinct yet complementary approaches developed to study the cognitive processes underlying judgment and choice: normative (prescriptive) and process (descriptive) models. Normative approaches focus on models, such as expected utility theory, that describe how decision makers, optimally, should make use of information. The standard procedure is to compare the decision maker's actual performance with an ideal. Process models, on the other hand, attempt to focus on how people actually do make decisions and process information. A fundamental principle of the later approach is that cognitive processes should be studied by collecting data during the decision process as often as possible (Ford, Schmitt, Schechtman, Hults, \& Doherty, 1989). These data include what information was examined, when, and in what order. This information then is used to derive a variety of process measures that enable researchers, among other things, to make inferences about the decision strategies that were employed in arriving at a particular decision.

The purpose of the present paper is to introduce MouseTrace, a new, computerized process-tracing methodology that facilitates research designed to monitor information acquisition and search behavior. Although both approaches can and have made use of such technology, it is the latter approach on which the paper primarily concentrates.

Initial development of MouseTrace was funded in part by start-up funds from the Faculty of Pharmacy and a Connaught Grant from the University of Toronto. The authors thank Wynstan Tong for his programming expertise. Without him, this project would have assuredly failed. J.S. is currently a medical student at McMaster University, Ontario, Canada. Correspondence should be addressed to J. D. Jasper, Department of Psychology, University of Toledo, 2801 W. Bancroft Street, Toledo, OH 43606 (e-mail: jjasper@utnet.utoledo.edu).

\section{WHY MONITOR INFORMATION SEARCH?}

There are at least three reasons that information acquisition and search should be monitored. First, many decisionmaking theories imply certain patterns of search. Therefore, examining search behavior can serve to evaluate and extend decision-making theory. The linear or additive model, for example, assumes that each dimension (or attribute) for a decision alternative is given a value and that the dimensions are combined additively to produce an overall value for each alternative; comparisons between alternatives are then based on these overall values, and the alternative with the greatest value is selected. Such a compensatory strategy would imply an interdimensionaland constant pattern of search. The elimination-by-aspects model, on the other hand, implies a noncompensatory, intradimensional strategy and a search pattern that may be variable. With elimination-by-aspects, the most important dimension is examined first, and all alternatives that do not meet a predetermined criterion are eliminated from consideration; the process is repeated until all but one of the alternatives is eliminated. Interestingly enough, researchers utilizing information-monitoring methods have found that individuals actually use both strategies in some situations. For example, when the decision is complex (e.g., the number of alternatives or attributes is large), simpler, noncompensatory strategies serve to narrow down the original set of options, whereas a compensatory evaluation of the remaining options then leads to a final choice (Payne, 1976; Payne, Bettman, \& Johnson, 1993).

Second, information acquisition and search data can provide unique insights into a number of variables that are known to affect decision making, as well as a variety of individual differences identified in psychology. For example, Payne et al. (1993) argued that decision makers are 
adaptive in the sense that they employ a wide variety of choice strategies contingent upon the decision task. Levin, Huneke, and Jasper (2000) have extended this research and have demonstrated that those most likely to exhibit adaptiveness are those that are high on need-for-cognition (see Cacioppo \& Petty, 1982, for a description). This suggests, then, that individual differences in cognitive style may predict individual differences in process data, which in turn may predict choice. However, further research needs to be conducted before definitive conclusions can be made.

Process data have also been collected to explain why decision makers treat missing information options differently from complete information options (Jasper, 2002b), an inclusionary task (one in which the decision maker chooses favorable options) differently from an exclusionary one (one in which the decision maker rejects unfavorable options; Jasper, 2002b; Levin et al., 2000; Levin, Jasper, \& Forbes, 1998), and a negatively framed attribute differently from a positively framed attribute (Jasper, 2002a).

Finally, understanding what type of information was attended to, exactly how much, when, and in what particular pattern can be useful from a practical standpoint. Johnson (1996) mentioned its utility in designing decision aids ranging from labels on products and packages to computerbased decision support systems (e.g., helping people choose health insurance policies). Such information can also serve as a useful catalyst for changing policy. For example, understanding how physicians make prescribing decisions or how health professionals make allocation decisions (e.g., organs for transplant) may lead to real-world change (e.g., new guidelines, legislation, and so forth), particularly if the choices currently being made are poor ones. Faced with constrained budgets and high consumer demand for knowledge, governments and granting agencies have begun to place increasing weight on applicationoriented research, and one might argue that the information gained from monitoring information acquisition and search behavior can be applied in a variety of direct and useful ways.

\section{CURRENT METHODOLOGIES}

Current information-monitoring methodologies include mechanical information boards, eye movement recording techniques, and computerized technologies, such as MouseLab, ComputerShop, and MouseTrace. Information boards represent one end of the spectrum and require subjects to pull cards out of envelopes (typically arranged in matrix form with alternatives across the top and attributes down the side or vice versa), turn them over, and then place them back in the appropriate envelopes, one at a time. Because of their inability to gather certain kinds of data, their potential for reactivity, and the development of newer, more sophisticated process-tracing methods, their popularity has waned. The monitoring of eye fixations via sophisticated photoelectric sensing devices anchors the other end of the spectrum. However, the complexity and expense of such equipment has severely limited its wide- spread use. Thus, the most commonly used informationmonitoring methods are computer adaptations of the information display board.

MouseLab, the first of these methods to be developed, uses computer graphics to display a matrix of information that is accessed with a computer-based pointing device known as the mouse. The initial appeal of MouseLab was threefold: (1) It reduced the reactivity created by mechanical versions of the information display board, (2) the methodology came close to the recording of eye movements in terms of speed and ease of acquisition, and (3) perhaps most important, it minimized instrumentation cost and the difficulty of use for both subject and experimenter. Thus, it was widely adopted by researchers in process tracing. Over time, however, other software systems were introduced in an effort to build upon the MouseLab tradition and provide more flexibility to its users. Two such systems are ComputerShop and MouseTrace.

ComputerShop (Huneke \& Huneke, 1999) presents decision makers with a Windows-based, pull-down menu environment and, in so doing, allows for significantly more product information to be available to subjects during the search task. Unlike MouseLab, it also allows the subject to make multiple responses in one or two stages, which facilitates the examination of consideration set formation (see Shocker, Ben-Akiva, Boccara, \& Nedungadi, 1991, for a review). This, in turn, may help to better monitor and understand the process decision makers use to arrive at a final choice. However, pull-down menus, like those used in ComputerShop, may lead decision makers to utilize different strategies than does a matrix environment. As a result, its comparability with MouseLab may be in question. However, this issue has yet to be addressed.

The other new system is MouseTrace. Like ComputerShop, MouseTrace was designed to accommodate significantly more information than MouseLab and to allow multiple responses, as well as multiple decision stages. With MouseTrace, however, the information is presented in an alternative-by-attributematrix, thereby making it directly comparable with MouseLab. Furthermore, whereas ComputerShop allows for up to two stages, MouseTrace allows for more than two and allows (if properly programmed) the decision maker to choose the number of stages he/she will use. Other qualities that distinguish it from MouseLab are (1) its ease of use for both subjects and experimenters, (2) its attractive visual appeal (primarily because it was written in Visual Basic, as opposed to MS-DOS), and (3) its uniqueness in terms of its features (e.g., it allows decision makers to operate under either inclusionary or exclusionary frames) and the data it records (e.g., it collects information related to set size, attribute importance, and real-time alternative selections and eliminations). ${ }^{1}$

\section{HARDWARE AND SOFTWARE REQUIREMENTS}

MouseTrace is a self-contained program written in Visual Basic (Version 5.0) and designed to run on an IBM 
PC-compatible computer using a Microsoft Windows platform (Windows 95 or higher). It requires a minimum of $32 \mathrm{MB}$ of RAM and an operating speed of at least $100 \mathrm{MHz}$. In terms of disk space, at least $30 \mathrm{MB}$ is recommended for software and data storage. Because of the visual nature of the program, a color monitor of $17 \mathrm{in.} \mathrm{or} \mathrm{more}$ is also recommended.

\section{GENERAL CAPABILITIES}

\section{An Overview}

Because MouseTrace operates within a Windows environment, the program does not require extensive computer or programming expertise. The user interface consists of a series of dialogue boxes that require simple maneuvers, such as clicking buttons, checking boxes, and typing text. The program operates on the principle of schemas, of which there are two types, text and matrix. A complete program (schema file) consists of a series of text and matrix schemas. Text schemas are used primarily to present instructions and other textual information to the subject before and after encountering a matrix of information. Matrix schemas are used to present the actual choice task in the form of an alternative-by-attribute table or grid.

When a set of options first appears on the screen, the values for each alternative-attribute combination are "hidden" behind boxes (or cells) of the resulting matrix. To open a particular box and examine the information, the decision maker has to move the cursor into the box. Depending on how the investigator has "programmed" the task, either the box may open and close automatically, or the subject may be required to open and close the box manually by clicking the mouse button. Subjects are usually asked to choose a single alternative after selecting as many information boxes as desired. However, subjects may also be prompted to make multiple selections through the use of discrete steps (or stages) to narrow the original set of options to a final choice. Among other benefits, this allows researchers to monitor changes in the relative importance or impact of different attributes during the course of a decision and then relate these changes to individual difference factors (see Levin \& Jasper, 1995, for a more detailed description of this procedure).

\section{Basic Features}

An experimental example will be used to help clarify MouseTrace's basic operation. Complete details of the experiment can be found in an earlier issue of the journal (Jasper \& Levin, 2001, Experiment 2). The task required students to "put themselves in the place of a college senior who was interested in going to graduate school and then decide which school or schools to apply to." Sixteen options were presented, and each was described by its tuition costs, its geographical location with respect to Toronto, its reputation, its selectivity in admitting applicants, the likeability of the potential advisor, the likeability of his/her ongoing research, the amount of stipend offered, and whether or not the GRE exam was required for admission.
The goal of the experiment was to assess whether asking subjects to narrow their options across successive stages alters one's final choice and the process by which one arrives at it. In the phased specified condition, subjects were required to narrow the original set of options to a specific a priori number in each of three stages. In the phased unspecified condition, subjects were allowed to narrow as quickly or as slowly as they desired across the three stages. In the unphased (or control) condition, subjects made a final choice without the imposition of stages.

Following an initial cover story and an introduction to MouseTrace and its features, subjects were presented with the matrix shown in Figure 1. Note that in this example, all eight attributes, but only nine options, are visible at any one time; the size of the cells can be controlled by the experimenter, but if there is too much information to fit on one screen, MouseTrace will automatically include a scroll bar.

To create a matrix like the one in Figure 1, one enters the File menu via the password-protected administrative mode and clicks on "New." A dialogue box appears, entitled "Create a Schema File." Here, the investigator will be required to input the name of the new schema file. Once the file name has been entered, another dialogue box (like the one shown in Figure 2) will appear, which serves as the initial set-up for each page. In this box, the investigator will be required to choose between a text or a matrix schema, decide whether he/she wishes to implement a timer (for a time pressure experiment) and, if so, which kind (analogue or digital), and decide whether he/she wants to implement guidance boxes, which provide help balloons to subjects during the course of an experiment.

If the investigator chooses to create a text page, he/she will immediately be given a "blank" screen on which the investigatorcan type whatever he/she wishes. Text screens are generally reserved for experimental instructions. If the investigator chooses to create a matrix schema, however, he/she will be required to provide the number of attributes and alternatives desired and the height and width (in units called twips) of the resulting information boxes. This will lead to a matrix screen where the boxes and the associated box coordinates are unveiled and where one can input the necessary information by typing directly over the coordinate information (see Figure 3).

Included on each matrix screen (in administrative mode) is a button labeled "Advanced," which when clicked, leads to the dialogue box shown in Figure 4. It is through this box that one decides whether subjects will include or exclude their options, whether stages will be utilized and, if so, how many, how many choices will be allowed/required per stage, how many information boxes can be opened at any one time, what kind of summary data will be required by the investigator, and whether instructions will be needed between successive stages and, if so, what those instructions will be. These features will be described in more detail below.

Cells. Here, the experimenter decides whether the boxes will open and close automatically (upon box entry 


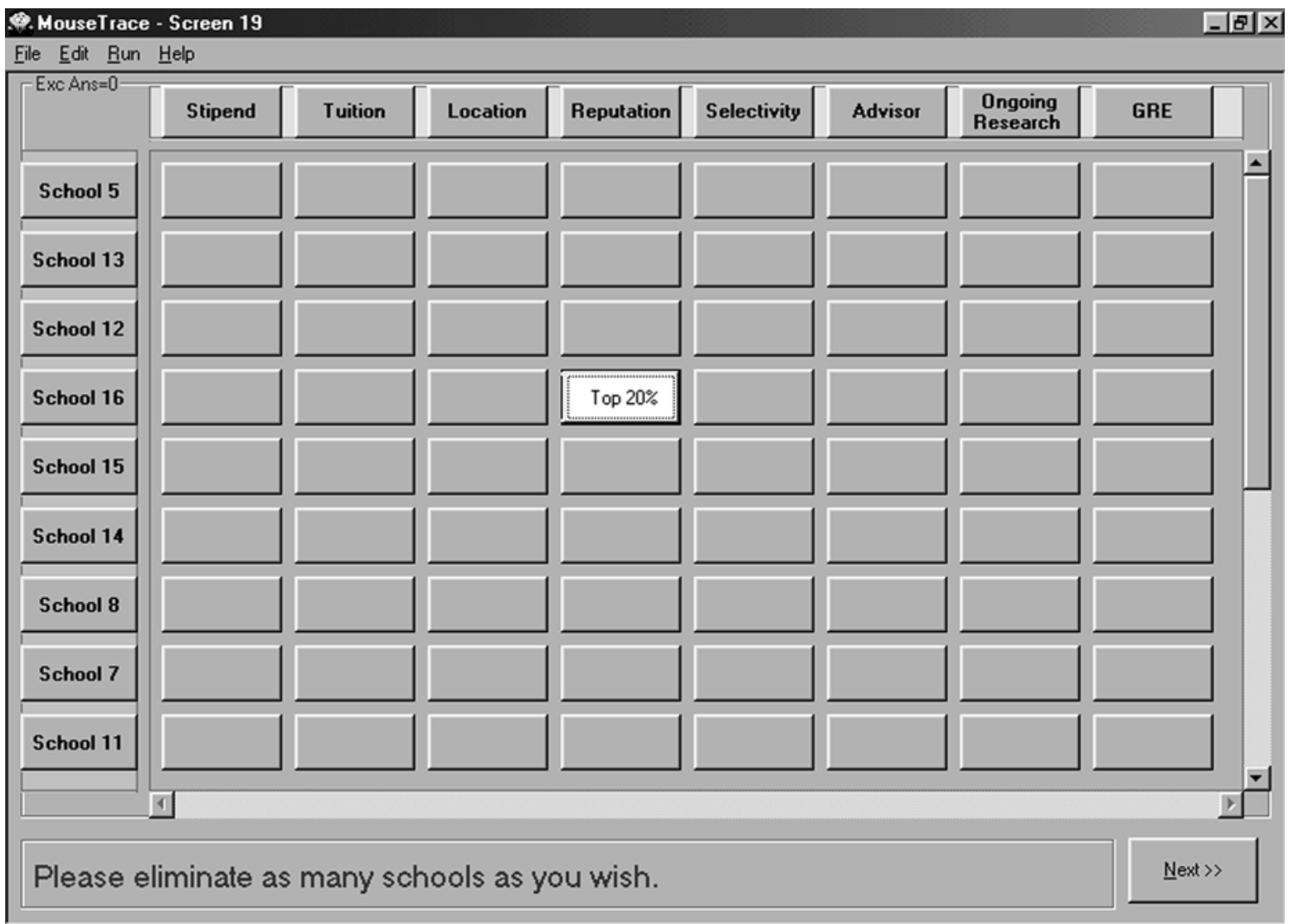

Figure 1. MouseTrace matrix screen, experiment (or "run") mode.

and exit) or manually (with a click of the left mouse button). The default is automatic, but in the graduate school example, the clicking method was chosen. Therefore, the small box to the left of this option was checked. In this section, one also indicates how many information boxes can be opened at any one time. In the graduate school study, the investigators believed it was necessary to restrict subjects' viewing to one box at a time (the default); thus, the

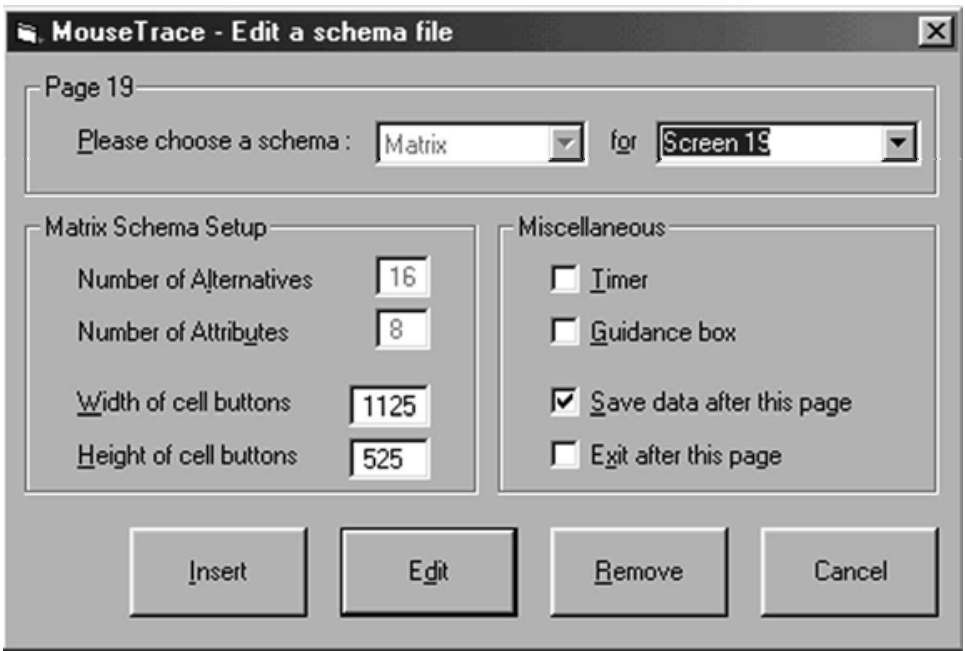

Figure 2. Initial set-up box for individual schem a pages. 


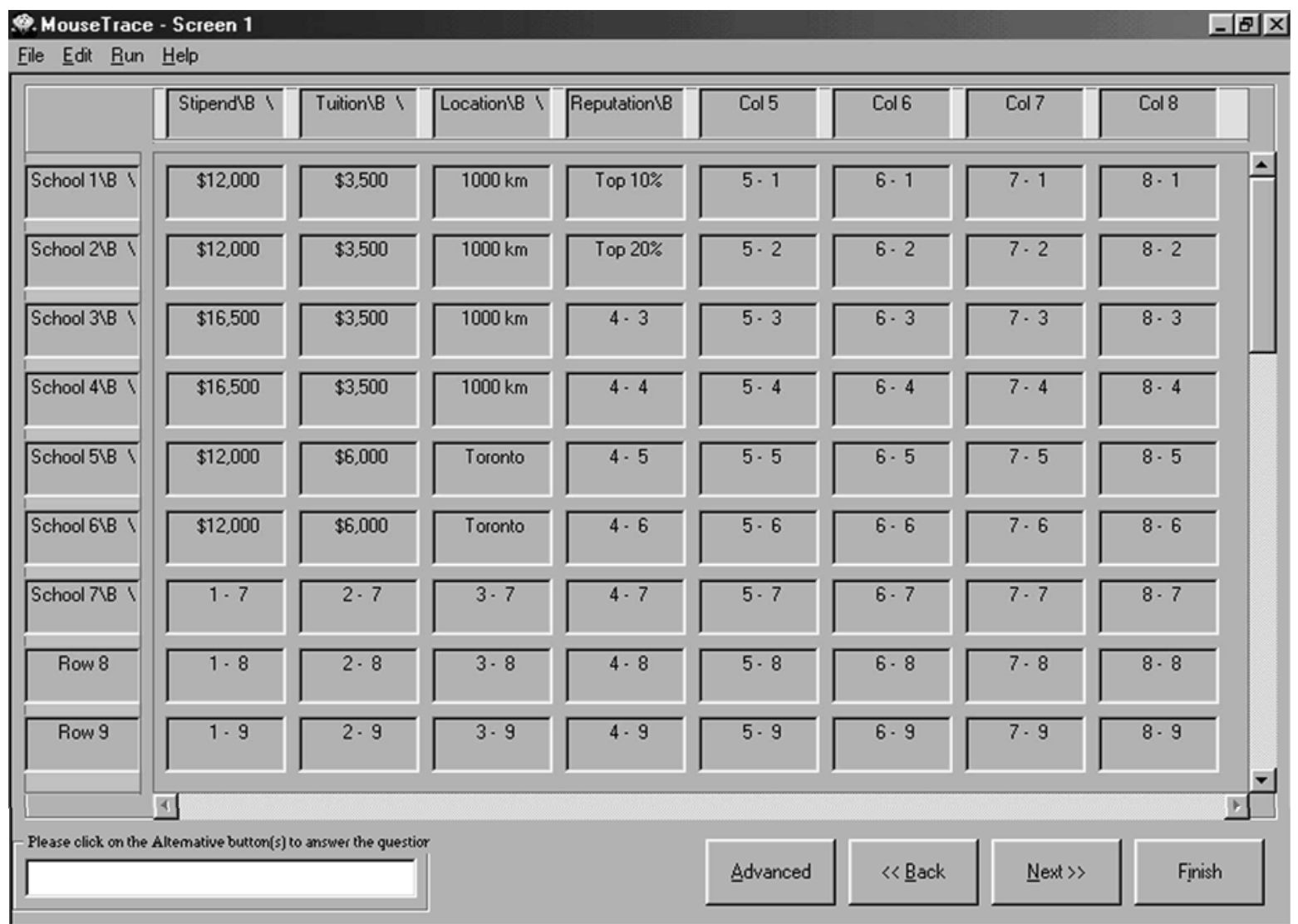

Figure 3. MouseTrace matrix screen, administrative mode.

small box in Figure 4 was left unchecked. However, in another study, it may be appropriate to allow subjects to see several boxes of information simultaneously; in this case, one checks the box to the left and then indicates the number of boxes that should remain open at any one time.

Inheritance stages. In the inheritance section, one decides whether to (1) incorporate stages in the decisionmaking task, (2) require a single or multiple response(s) and, if multiple, specify exactly how many responses, and (3) frame the task as one of either inclusion or exclusion of alternatives. All three features were utilized in the graduate school study. ${ }^{2}$

This section is also where the experimenter specifies how much time (in seconds) to give subjects in a time pressure experiment and what message to give subjects when time is up (see time-out). Finally, this section is used to decide whether instructions will be needed between successive stages and, if so, what those instructions will be. These instructions are placed in what are labeled confirmation screens (or dialogue boxes), which are also capable of listing the alternatives that have been selected or eliminated by the subject in each stage.

Figure 5 shows a confirmation screen from the graduate school study. If the subjects are satisfied with their selections, MouseTrace will recompile the remaining alter- natives and present the subjects with another matrix of information (i.e., a second stage); the process continues until all stages have been exhausted and/or a final choice has been made. If the subjects are unhappy with their choices, the program allows them to return to the original matrix to make the needed changes. Selections (and deselections) are made on the matrix screens themselves by moving to the far left side of the screen and clicking on the alternative names. If an alternative is selected, the alternative button will turn from gray to red (and back again, if deselected).

Data options and analyses. MouseTrace is designed to record a variety of temporal data, and it does this automatically. However, the data is very much on line and fairly raw and is provided in such a way that necessitates a bit of filtering to obtain useful information. Therefore, the Data Options and Analyses section filters (or summarizes) some of the more useful numbers. Currently, these summary data include (1) the times that the alternatives were selected (and deselected), (2) the time differences between the closing of one box and the opening of another, (3) the time differences between entry into a box and the opening of that box (of course, this is useful only in tasks in which the subject must click to open and close boxes), (4) the time differences between the opening and 


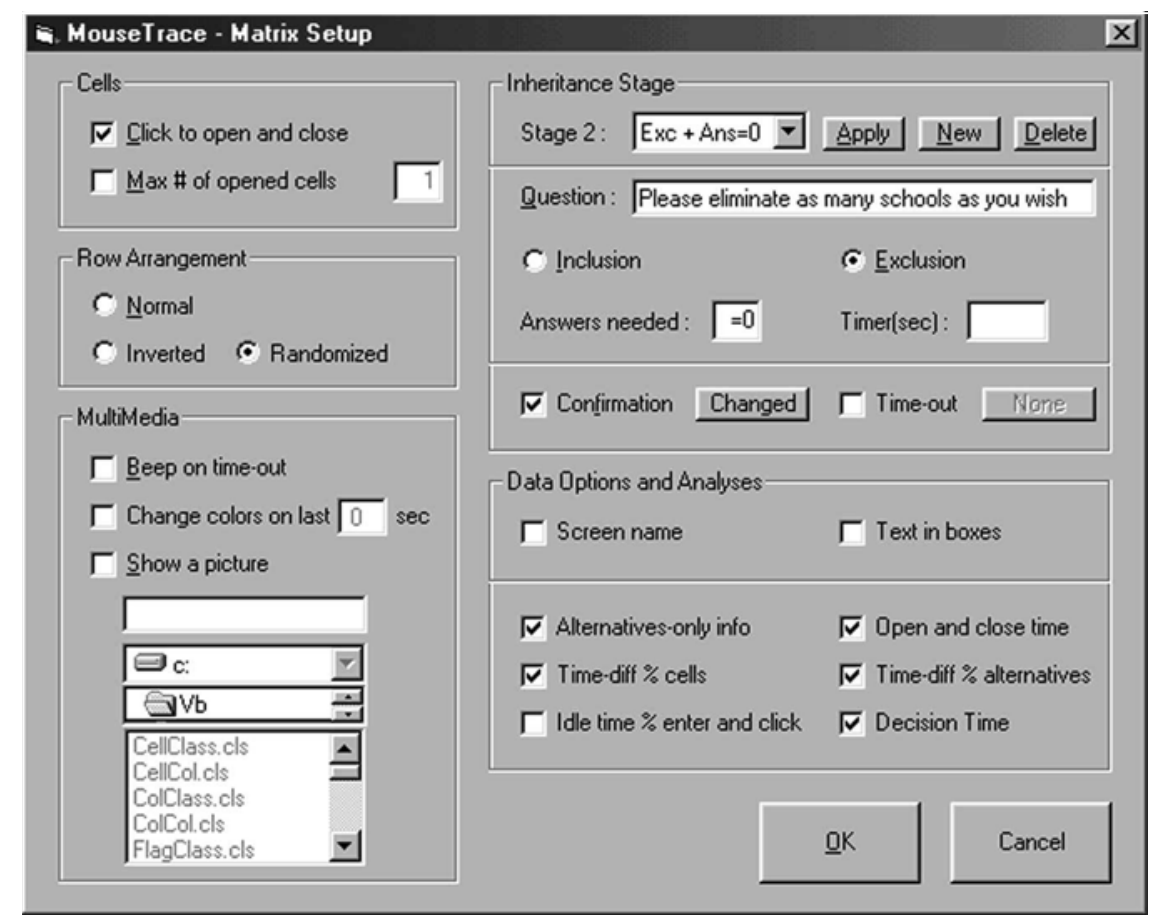

Figure 4. “Advanced” features set-up box.

closing of the same box, (5) the time differences between the selection of one alternative and the selection of another, and (6) the total decision time for each matrix screen (calculated from the moment the start button is clicked to the moment the next button is pressed). To select any of these summary measures, the experimenter need only check the appropriate boxes to the left of the corresponding options. In the graduate school study, all but No. 3 were checked.

\section{Other Features}

Mouse Trace has two other main features. Row arrangement, as the name implies, allows the experimenter to choose the order in which he/she wants the alternatives to appear. "Normal" indicates the order in which the alternatives were initially typed, "inverted" is the reverse, and "random" randomizes the alternatives. The program also enables the investigator to substitute graphics (icons, pictures, etc.) for text; the multimedia section is particularly applicable

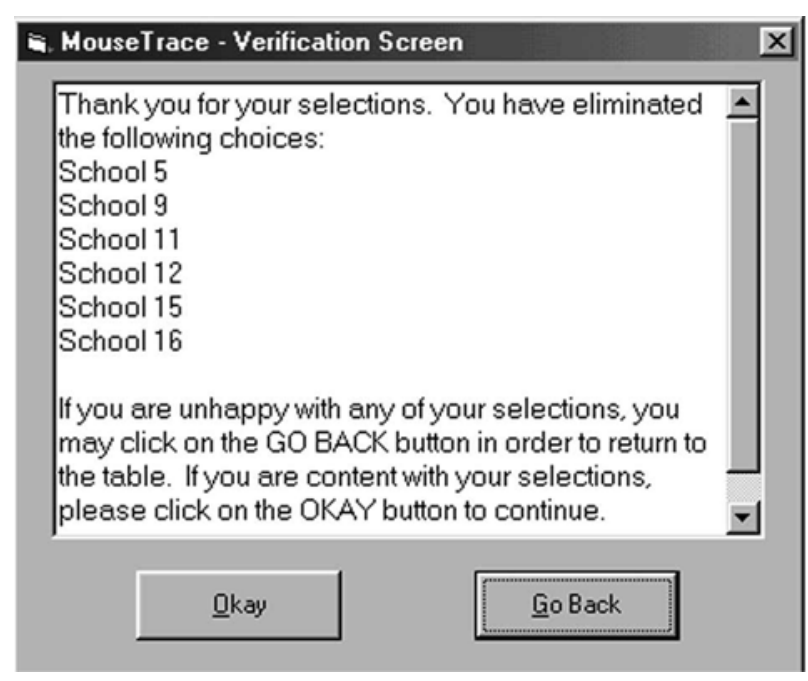

Figure 5. Confirmation (or verification) screen. 
in product-packaging experiments. It also allows the investigator to provide additional sensory cues in time pressure experiments. "Change colors," for example, lets subjects know that time is almost up, whereas "beep on time out" alerts subjects to the fact that time has indeed run out. In the graduate school study, the rows (or alternatives) were randomized, and there was no need for multimedia.

\section{The Output}

In terms of actual data, Mouse Trace records what boxes were opened and closed, when (measured to one thousandth of a second), and in what order. It also indicates the alternatives chosen at each step (or stage), the time at which they were chosen, the order in which they were selected and/or deselected, and the total decision time for each stage and the entire task. MouseTrace creates a log file for each subject, which can be accessed through Notepad in the Windows application (see Figure 6 for an example). These data, in concert with the summary measures mentioned previously (see Figure 7 for an example), are used to derive a variety of process measures, including measures related to what process researchers refer to as depth, sequence, and content. ${ }^{3}$

Depth measures. These measures refer to the amount of information accessed from the available information environment; these are related to effort and are used in conjunction with sequence measures to determine decision strategies. Useful measures include (1) the total number of information items accessed, (2) the proportion of information accessed, (3) the number of options or attributes considered, (4) the proportion of options or attributes considered, (5) the decision time, and (6) the average time spent per acquisition.

Sequence measures. These measures generally refer to the temporal pattern in which information was acquired and assessed through a comparison of the $n$th and the $n$th +1 pieces of information searched. They include (1) the proportion of Types 1 (same-option-same-attribute), 2 (same-option-different-attribute), 3 (different-optionsame-attribute), and 4 (different-option-different-attribute) transitions that occur, (2) the variance in the proportion of information searched per option or attribute, (3) the variance in the proportion of time spent on each option or attribute, and (4) "search pattern," an index (originally developed by Payne, 1976, and his associates and later modified by Bockenholt \& Hynan, 1994) used to describe whether information selection behavior was mostly attribute or alternative based.

Content measures. These measures refer to exactly what information was acquired and which option(s) were chosen. Indices include (1) the proportion of times a given option or attribute was acquired, (2) the proportion of time

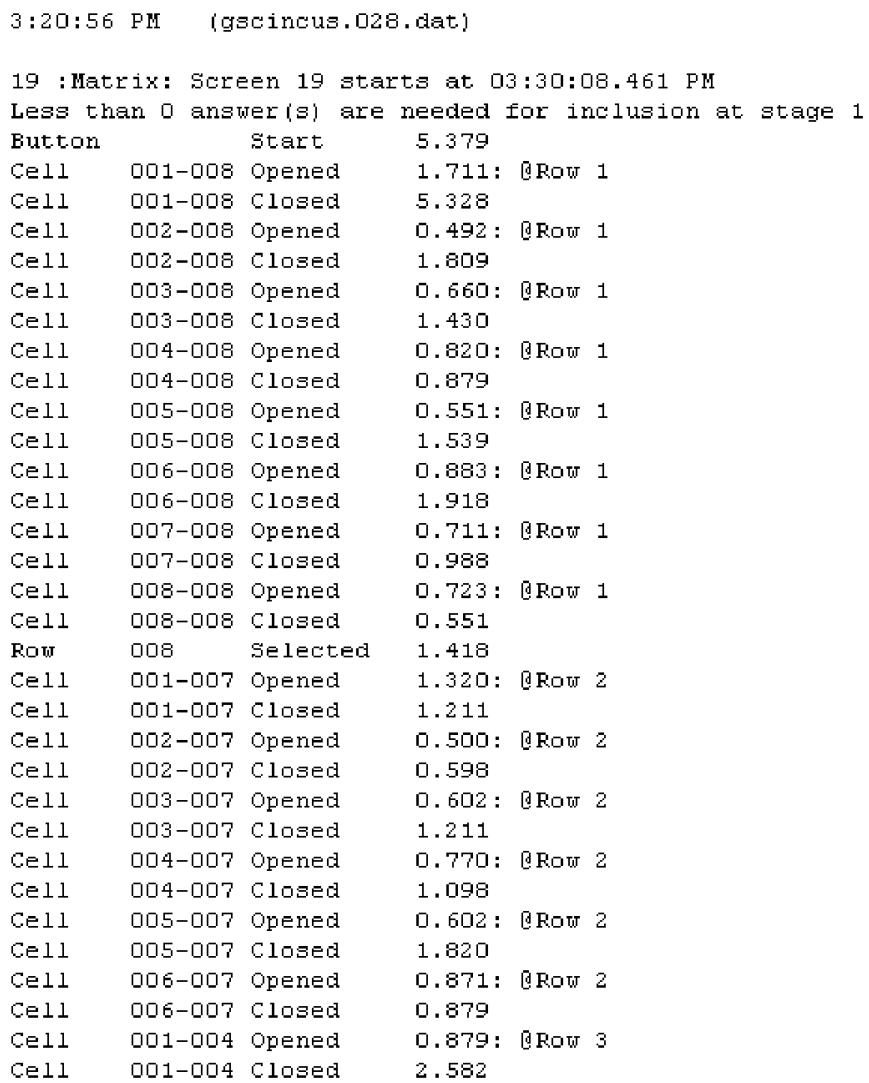

Figure 6. Sample subject data (raw). 


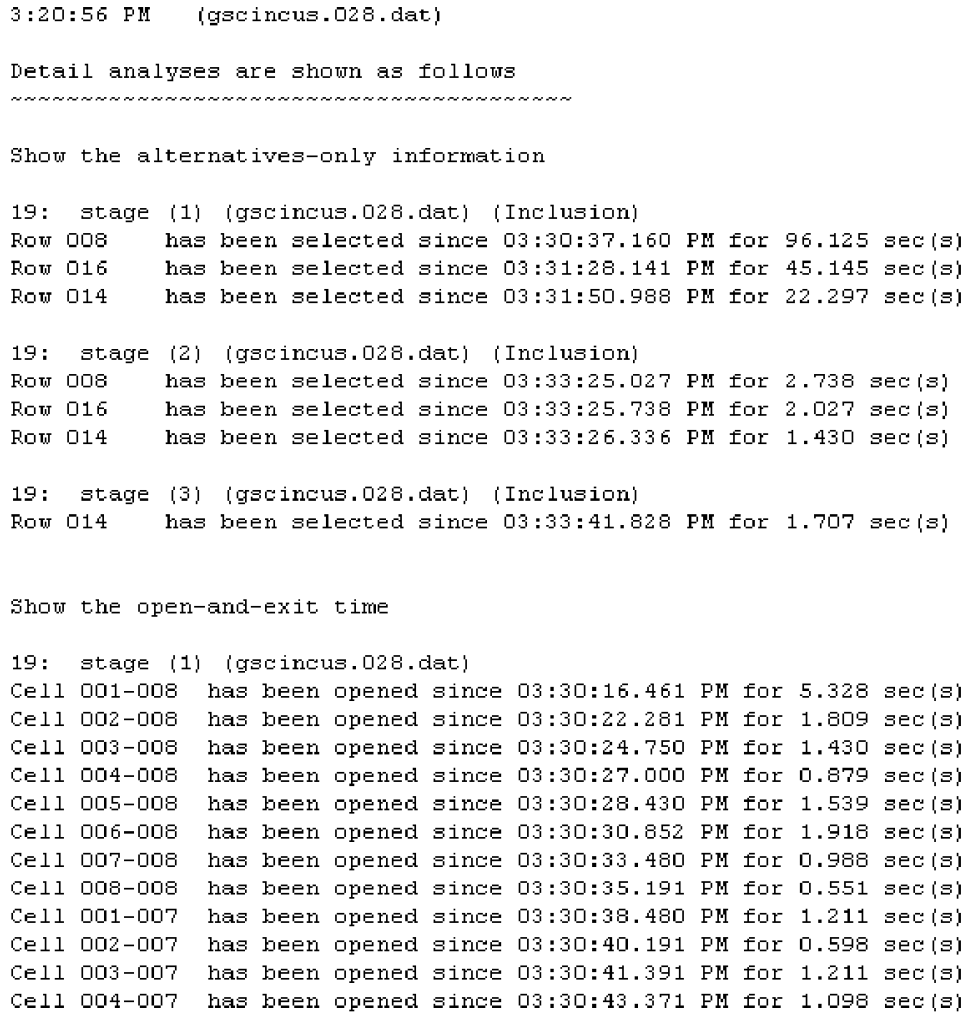

Figure 7. Sample subject data (summary).

spent on each attribute or option, (3) the proportion of subjects considering a given option or attribute, (4) the relative importance of each attribute as assessed through attribute values, and (5) the distribution of options chosen.

The above measures are not exhaustive, but they should give one an idea of the kind and diversity of indices that can be extracted from a process approach. The vast majority of these measures are interval or ratio in nature and, therefore, they can be subjected to parametric statistical techniques.

The data from MouseTrace can be imported directly into such programs as SAS and SPSS. However, a companion data analysis program has been developed and is available from the first author. The program has been written in Microsoft Excel and converts MouseTrace's summary data into a variety of process indices (see Figure 8 for an example of process output). These process measures can then be entered into SAS or SPSS for formal statistical analysis.

In the graduate school study, the primary focus was to assess the impact of stages on the processes involved in such a task. Therefore, the emphasis was placed on overall and stage-related measures of information processing, such as total number of acquisitions, total search time, attribute impact, search pattern, and the distribution of choices. ${ }^{4}$ For those who are curious, the results revealed that there were no differences between the three conditions (phased specified, phased unspecified, and unphased/ control) with respect to the distribution of final choices. However, there were many differences between the conditions in terms of traditional process variables, and the vast majority of the evidence singled out the phased specified condition as being different from the other two conditions. Specifically, the subjects in the phased specified condition spent more time looking at information and making their final decisions, made a greater number of acquisitions (i.e., looked at more information), searched that information with greater breadth and depth, and probed more deeply into each of the attributes. In addition, although all three conditions demonstrated a decrease in decision time, open box time, and time spent per attribute examined (depth of search) across time, these measures decreased more rapidly across stages in the phased specified condition than in the other two conditions. Therefore, the investigators concluded that if one wants to use phased narrowing to study decision making, allowing decision makers to determine their own set sizes en route to making a final decision is the method of choice.

\section{OTHER APPLICATIONS}

The graduate school choice study is but one example of a number of decision contexts. Product selection, of course, is a natural for this type of methodology. The first author 


\begin{tabular}{|c|c|c|c|c|c|c|c|c|c|}
\hline Partici & t Code & & & ic001 & ic002 & ic003 & ic004 & ic006 & ic007 \\
\hline Total T & spent & & & 913.746 & 406.465 & 641.375 & 376.691 & 350.836 & 175.086 \\
\hline Stage & & & & 0 & 0 & 0 & 0 & 0 & 0 \\
\hline Option & Iternatives & & & & & & & & \\
\hline Numb & f Rows & & & 16 & 16 & 16 & 16 & 16 & 16 \\
\hline Numb & f Rows Exa & Imined & & 16 & 16 & 16 & 16 & 16 & 16 \\
\hline Attribu & /Characteris & stics & & & & & & & \\
\hline Numbe & f Columns & & & 8 & 8 & 8 & 8 & 8 & 8 \\
\hline Numbe & f Columns & Examined & & 8 & 8 & 8 & 8 & 8 & 8 \\
\hline & & & & & & & & & \\
\hline \# of ac & sitions = & & & 444 & 168 & 145 & 168 & 167 & 71 \\
\hline \# of tra & tions = & & & 443 & 167 & 144 & 167 & 166 & 70 \\
\hline Total ti & spent $=$ & & & 913.746 & 406.465 & 641.375 & 376.691 & 350.836 & 175.086 \\
\hline Total P & ible boxes & & & 128 & 128 & 128 & 128 & 128 & 128 \\
\hline Total \# & new boxes & opened & $X X$ & 88 & 104 & 91 & 121 & 75 & 47 \\
\hline Propor & lof Informa & tion Accesse & & 0.6875 & 0.8125 & 0.710938 & 0.945313 & 0.585938 & 0.367188 \\
\hline Total 0 & box time & $(\mathrm{OBT})=$ & $X$ & 377.719 & 166.779 & 328.807 & 143.537 & 171.514 & 57.433 \\
\hline Averag & otal Time S & pent $/ A c q=$ & & 2.057986 & 2.419435 & 4.423276 & 2.242208 & 2.100814 & 2.466 \\
\hline Averag & BT Spent / & $A c q=$ & & 0.850718 & 0.992732 & 2.267634 & 0.854387 & 1.02703 & 0.808915 \\
\hline & & & & & & & & & \\
\hline Breadt| & Search (\# & Attr/Alt $)=$ & & 5.5 & 6.5 & 5.6875 & 7.5625 & 4.6875 & 2.9375 \\
\hline Attribu & robe (\# Ac & qu/Attrib) $=$ & & 55.5 & 21 & 18.125 & 21 & 20.875 & 8.875 \\
\hline Deptho & $\operatorname{arch} 1(\mathrm{OB})$ & T/Alt examin & ned) $=$ & 23.60744 & 10.42369 & 20.55044 & 8.971063 & 10.71963 & 3.589563 \\
\hline Deptho & $\operatorname{arch} 2(\mathrm{OB}$ & T/Attrib exan & mined) $=$ & 47.21488 & 20.84738 & 41.10088 & 17.94213 & 21.43925 & 7.17 \\
\hline
\end{tabular}

Figure 8. Sample process data from the graduate school study.

(Jasper, 2002a), in fact, has recently collected data for a project in which physicians are compared with pharmacists as to how they make recommendations to hypothetical patients/clients about over-the-counter (OTC) medications. The same researcher (Jasper \& Tam, 2000) has also recently investigated the lay public's choice of OTC products (e.g., sunscreens) and how various pharmacistdelivered pharmaceutical care interventions might improve these decisions.

Person selection is also a possibility (e.g., applicants to hire for a job, recipients to select for an organ transplant). Skitka, Jasper, and Mullen (2002), for example, are currently testing whether the decision processes that sometimes produce tragic choices in resource allocation contexts are consistent with the four-stage contingency model of distributive justice (Skitka \& Tetlock, 1992). Specifically, these investigators are using MouseTrace to explore (1) the decision-making processes that underlie allocation choices in health care domains (organs for transplant and AZT for AIDS victims) under conditions of scarcity and no scarcity and (2) the generalizability of the model, particularly the importance assigned to personal responsibility information, by comparing choices made by individuals across two very different health care delivery systems: the free market system of the United States and the universal care system of Canada.

In sum, MouseTrace works well in any complex environment in which information can be presented in a grid- like pattern and choices can be made. The environments within which decision researchers typically work are likely to be akin to those mentioned above. However, other practical and theoretical contexts can be envisioned as well. For example, as was indicated earlier, one could easily imagine MouseTrace being used to explore a variety of issues in areas such as product packaging and policy. Although it may be a bit more of a stretch, one might also envision MouseTrace being utilized to better understand impression formation, object categorization, and associative learning, among others.

The possibilities indeed appear promising. However, the reader should be cautioned. It is not being suggested that MouseTrace can be used in every situation. There may be circumstances, for instance, that do not lend themselves to a matrix environment. There may also be theories, individual differences, or variables that cannot be evaluated and/or interpreted using information search and acquisition data. Decision-making theories, for example, tend to lie along a continuum from analytic to nonanalytic approaches. As one might guess, MouseTrace is better suited for analytical models that assume a decision maker who actively combines or processes information mentally. A good example of such a model was proposed by Payne and his associates (Payne et al., 1993), who focused on the role played in strategy choice by the cognitive (or computational) effort required to execute a strategy in a specific task environment. 
Hastie and Pennington (2000), at the other end of the continuum, proposed a mental model of decision making that synthesizes the rule-based (Rips, 1994) and mental models (Johnson-Laird, 1983) views of reasoning. According to their explanation-based (or story) approach, decision makers begin their decision process by constructing a causal model to explain the available facts. A decision is made when the causal model of the evidence is successfully matched to an alternative in the choice set. Such an approach is extremely valuable to an understanding of decision making, but it is difficult (if not impossible) to capture within the theoretical and programming confines of MouseTrace.

There also exists a third class of theories that take a more middle-of-the-road stance by combining the best features of both analytical and nonanalytical models. Image theory (Beach, 1990, 1993; Beach \& Mitchell, 1987) is a good example. Image theory suggests that there are two kinds of decisions, adoption and progress decisions. Adoption decisions involve the adoption or rejection of candidate goals or plans as constituents of what are referred to as the trajectory or strategic images. Progress decisions consist of assaying the compatibility (or fit) between the forecasted future if a given plan is implemented (or continues to be implemented) and the ideal future as defined by the trajectory image. Incompatibility triggers rejection of the plan and adoption of a substitute. Failure to find a feasible substitute prompts reconsideration of the plan's goal.

Adoption decisions involve screening and choosing alternatives and, thus, with a few programming changes, would pose little to no problem for MouseTrace. Progress decisions, on the other hand, like explanation-based approaches, would probably be impossible for MouseTrace to evaluate. So, in essence, MouseTrace can be used to evaluate some, but not all, elements of image theory, and those elements that can be successfully evaluated tend to be more analytical in nature.

\section{ONGOING DEVELOPMENTS}

Although the program has already provided a number of insights into human behavior and is quite usable in its current form, the development of MouseTrace is ongoing. Other features that may be added in the near future are allowing (1) the experimenter to manipulate the order of attributes, (2) the experimenter to add, subtract, or change information between stages, (3) decision makers to start over if options become "unavailable" (see Potter \& Beach, 1994), and (4) decision makers to stop between stages for time lag experiments. The biggest enhancement, though, may be in MouseTrace's accessibility. Subjects in previous MouseTrace studies have included professionals, such as physicians, pharmacists, and health policy makers, who find it difficult either to come to the lab or to schedule a specific block of time in which they are free. For experimenters, it is also inefficient and potentially expensive (if not impossible, particularly with a national sample) to track down such subjects. Thus, the long-term goal is to make the program itself and the experiments, in particular, available through the World-Wide Web. The Internet should not only make MouseTrace more accessible, but also provide the impetus for using the program as a computerized decision aid.

\section{DISTRIBUTION POLICY}

The current version of Mouse Trace is available on platforms running Windows 95 or higher. A Mac version will most likely not be developed; however, PC emulation software and PC cards for the Mac are available and can be used to circumvent this problem. The Web version, as was mentioned, is still in the planning phases. MouseTrace is available for a small development and handling fee of $\$ 30$ U.S. Requests should be made in writing to J. D. Jasper, Department of Psychology, University of Toledo, $2801 \mathrm{~W}$. Bancroft Street, Toledo, OH 43606 or by e-mailing Dr. Jasper at jjasper@utnet.utoledo.edu. All correspondence regarding MouseTrace, including bug reports, questions, and suggestions, should be sent to the same address.

\section{REFERENCES}

BEACH, L. R. (1990). Image theory: Decision making in personal and organizational contexts. Chichester, U.K.: Wiley.

BEACH, L. R. (1993). Image theory: Personal and organizational decisions. In S. A. Klein, J. Orasanu, R. Calderwood, \& C. E. Zsambok (Eds.), Decision making in action: Models and methods (pp. 148157). Norwood, NJ: Ablex.

Beach, L. R. \& Mitchell, T. R. (1987). Image theory: Principles, goals, and plans in decision making. Acta Psychologica, 66, 201-220. Bockenholt, U., \& HyNAN, L. S. (1994). Caveats on a process tracing measure and a remedy. Journal of Behavioral Decision Making, 1, 103-117.

Cacioppo, J. T., \& Petty, R. E. (1982). The need for cognition. Journal of Personality \& Social Psychology, 42, 116-131.

Ford, J. K., Schmitt, N., Schechtman, S. L., Hults, B. M., \& DoHerty., M. L. (1989). Process tracing methods: Contributions, problems, and neglected research questions. Organizational Behavior \& Human Decision Processes, 43, 75-117.

Hastie, R., \& Pennington, N. (2000). Explanation-based decision making. In T. Connolly, H. R. Arkes, \& K. R. Hammond (Eds.), Judgment and decision making: An interdisciplinary reader (pp. 212-228). New York: Cambridge University Press.

Huneke, M. E., \& Huneke, A. C. (1999). ComputerShop: The next generation in information search monitoring technology. Unpublished manuscript, University of Iowa.

JASPER, J. D. (2002a). The effects of illness severity and information frame on medication decisions: Insights from process tracing. Manuscript in preparation.

JASPER, J. D. (2002b). How decision makers deal with missing information. Manuscript in preparation.

JASPER, J. D., \& LEVIN, I. P. (2001). Validating a new process tracing method for decision making. Behavior Research Methods, Instruments, \& Computers, 33, 496-512.

JASPER, J. D., \& TAM, R. (2000, November). The effects of pharmaceutical care on sunscreen decisions. Paper presented at the annual meeting of the Society for Judgment and Decision Making, New Orleans. Johnson, E. J. (1996). The last whole MouseLab manual (Version 6.0). Available from http://www-marketing. wharton.upenn.edu/ mouselab/. JOHNSON-LAIRD, P. N. (1983). Mental models: Towards a cognitive science of language, inference, and consciousness. Cambridge, MA: Harvard University Press.

Levin, I. P., Huneke, M. E., \& JASPER, J. D. (2000). Information pro- 
cessing at successive stages of decision making: Need for cognition and inclusion-exclusion effects. Organizational Behavior \& Human Decision Processes, 82, 171-193.

LEVIN, I. P., \& JASPER, J. D. (1995). Phased narrowing: A new process tracing method for decision making. Organizational Behavior \& Human Decision Processes, 64, 1-8.

LeVIN, I. P., JASPER, J. D., \& Forbes, W. S. (1998). Choosing versus rejecting options at different stages of decision making. Journal of Behavioral Decision Making, 11, 193-210.

PAYNe, J. W. (1976). Task complexity and contingent processing in decision making: An information search and protocol analysis. Organizational Behavior \& Human Performance, 16, 366-387.

Payne, J. W., Bettman, J. R., \& Johnson, E. J. (1993). The adaptive decision maker. Cambridge: Cambridge University Press.

Potter, R. E., \& BeACH, L. R (1994). Decision making when the acceptable options become unavailable. Organizational Behavior \& Human Decision Processes, 57, 468-483.

RIPS, L. J. (1994). The psychology of proof: Deductive reasoning in human thinking. Cambridge, MA: MIT Press.

Shocker, A. D., Ben-Akiva, M., Boccara,B., \& Nedungadi,P. (1991). Consideration set influences on consumer decision-making and choice: Issues, models, and suggestions. Marketing Letters, 2, 181-197.

SkitKa, L. J., JASPER, J. D., \& Mullen, E. (2002). Understanding ideological differences in public compassion: A process tracing approach. Submitted for publication.
SkIT KA, L. J., \& TETLOCK, P. E. (1992). Allocating scarce resources: A contingency model of distributive justice. Journal of Experimental Social Psychology, 28, 491-522.

\section{NOTES}

1. Some might argue that the functionality of MouseTrace could easily be implemented in Mel, PsyScope, E-Prime, or some other experimentgenerating environment that may afford more design flexibility and facilitate data acquisition. This could well be true. However, MouseTrace provides a self-contained, integrated environment in which the informationchoice paradigm can be efficiently used to study decision making across a variety of contexts.

2. Complete details for "programming" the inheritance section can be found in the MouseTrace manual, which is available upon request.

3. MouseTrace creates an Excel version of the summary data (in addition to the Notepad version) which can be used for subsequent data analysis.

4. For empirical data, please refer to Tables 7 and 8 of Jasper and Levin (2001).

(Manuscript received June 20, 1999;

revision accepted for publication March 18, 2002.) 